\title{
effet d'échelle et surfaces de glissement
}

\author{
P. HABIB \\ Laboratoire de mécanique des solides \\ (Ecole Polytechnique* - E.N.S.M. Paris - \\ E.N.P.C. - Laboratoire Associé au C.N.R.S.)
}

\section{Résumé}

Le développement progressif d'une surface de glissement localisée dans les sables denses présentant du radoucissement est responsable de l'effet d'échelle que différents auteurs ont constaté pour des poinçons de moins de $15 \mathrm{~cm}$ de large. II n'y a pas d'effet d'échelle pour les poinçons sur sables lâches ni sur les argiles moyennement plastiques. II en existe probablement un pour les argiles très raides.

Des angles de frottement interne extrêmement grands peuvent être mis facilement en évidence sur des sables secs dans un équilibre de Rankine. Ils sont à rapprocher de ce que l'on observe avec les massifs d'enrochements compactés utilisés pour les barrages.

\section{Abstract}

The progressive development of a slip surface (localisation) in dense sands with softening is responsible for the scale effect observed by various authors for foundations smaller than $B=15 \mathrm{~cm}$. There is no scale effect for foundations on loose sands or on soft clays. There is probably a scale effect for stiff clays.

Very great angles of internal friction can be obtained with Rankine's equilibrium for dry sands. This is very similar to what can be observed with compacted rockfills for dams. 


\section{INTRODUCTION}

Il est bien connu que les poinçonnements des petites fondations sur des sables denses donnent des forces portantes relativement plus grandes que celles qu'on observe avec les grandes fondations. Pour être plus précis, si on écrit la valeur de la pression limite $\mathrm{q}_{\mathrm{u}}$ sous la forme classique $\mathrm{q}_{\mathrm{u}}=\frac{1}{2} \gamma \mathrm{B} \mathrm{N}_{\gamma}$ le coefficient de portance $N_{\gamma}$ est très grand pour les petits modèles (P. HABIB, 1961; E.E. DE BEER, 1965; S. AMAR et al., 1984).

On n'a rien constaté d'analogue jusqu'à présent pour les poinçonnements sur argile, et le terme de cohésion $\mathrm{CN}_{c}$ semble indépendant de la dimension de la fondation. Ce phénomène pour les sables a été appelé "effet d'échelle "sans qu'on en comprenne clairement la cause. Il en est résulté une certaine méfiance vis-à-vis des essais sur modèles réduits sur sables secs et aussi d'ailleurs vis-à-vis des formules théoriques.

Une tentative d'explication de cet effet d'échelle a été apportée en remarquant que la dilatance de la surface de glissement (c'est-à-dire en fait la dilatance de ce volume composé de quatre à cinq couches de grains qu'on appelle la surface de glissement) était la même pour un petit modèle que pour un grand. La dilatance d'une surface de glissement est évidemment fonction de la dimension des grains. La dilatance ne respecte donc pas la similitude si deux fondations homothétiques sont placées sur le même sable (fig. 1).

Dans un tel cas l'importance relative de la dilatance dans le travail de déformation correspondant au poinçonnement est plus grande pour les petits modèles que pour les grands, d'où des forces portantes plus grandes que ce que l'on pouvait prévoir (P. HABIB, 1973).

L'explication précédente paraît cependant insuffisante pour deux raisons:

d'une part, elle n'est valable que s'il n'existe qu'une seule surface de glissement; or, l'expérience montre qu'après l'apparition de la première surface, des ruptures secondaires se produisent dessinant finalement un réseau compliqué de surfaces de glissement, et cela aussi bien sous un poinçon dans une caisse de sable au laboratoire que pour un glissement de coteau dans la nature; mais, d'autre part, l'explication de l'effet d'échelle par la dilatance d'une surface de glissement serait en défaut si le soulèvement du sol lié à la dilatance, puis le phénomène de rupture générale se produisaient l'un après l'autre. Cette objection m'avait été faite en son temps par le Professeur Jean MANDEL, au cours d'une conversation personnelle. Je vais montrer que la progression de la surface de glissement et l'augmentation de sa longueur avec l'augmentation du chargement, alliées à la notion de rupture progressive associée à la formation d'une surface de glissement dans un milieu radoucissant (P. HABIB, 1984), permettent de compléter l'interprétation précédente.

\section{EFFET D'ÉCHELLE DANS LE POINÇONNEMENT DES SABLES SECS}

Il est bien clair que lorsqu'une fondation est suffisamment grande pour que la surface de rupture dans le sable progresse avec l'augmentation du chargement, le travail de la dilatation est en quelque sorte étalé sur toute la durée du chargement. La rupture progressive conduit alors à étudier l'équilibre final sur une surface de glissement où la résistance au cisaillement correspond à l'angle de frottement résiduel même si l'orientation initiale et la forme de cette surface sont des fonctions de $\varphi_{\max }$ (ou de la dilatance associée à $\varphi_{\max }$ ).

Il est d'autre part classique dans les essais de cisaillement à la boîte de Casagrande, d'observer qu'un déplacement relatif de 3 à $5 \mathrm{~mm}$ est nécessaire pour atteindre puis dépasser le maximum de résistance et pour que le plan de glissement soit complètement développé entre les deux demi-boîtes. De même, pour un essai triaxial standard avec des éprouvettes de sable dense de $35 \mathrm{~mm}$ de diamètre le plan de glissement apparaît après un déplacement relatif des bases de l'échantillon du même ordre de grandeur et le glissement entre les parties d'apparence monolithique de chaque côté de la surface de glissement est encore du même ordre de grandeur.

Enfin, c'est un autre résultat classique pour le poinçonnement des fondations, de constater que la rupture est consommée lorsque l'enfoncement $w$ atteint $5 \%$ de la largeur B de la base (fig. 2).
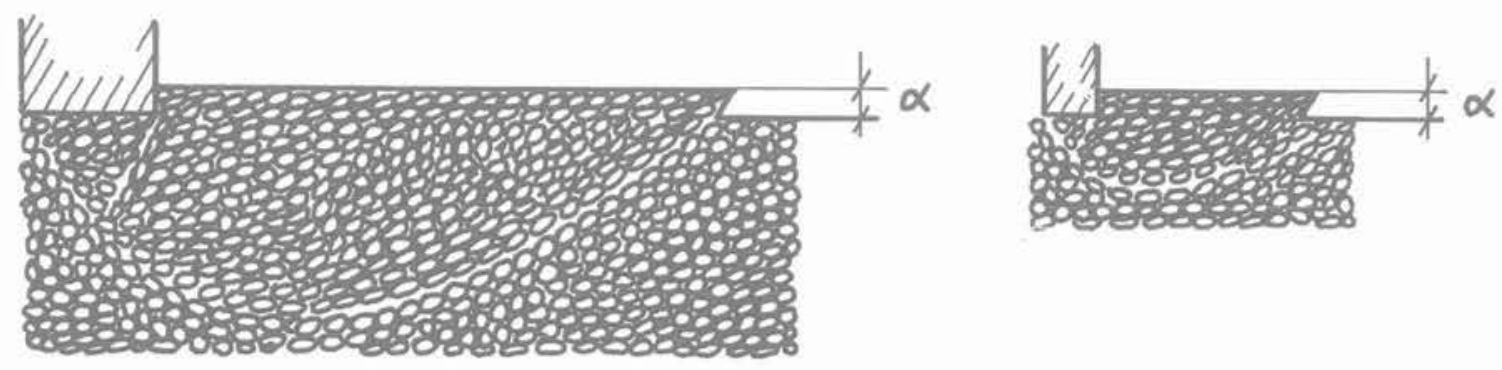

Fig. 1. - La dilatance de la surface de glissement a la même amplitude $(\alpha)$ pour deux fondations homothétiques placées sur le même sable. 

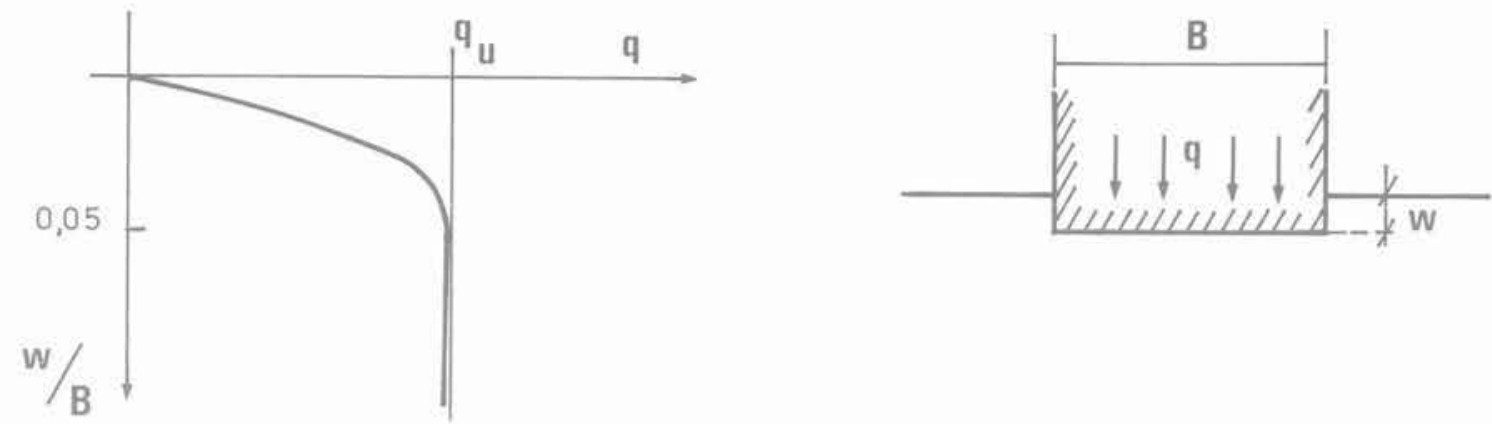

Fig. 2. - Diagramme classique du poinçonnement d'une fondation.

Le rapprochement de ces deux grandeurs:

3 à $5 \mathrm{~mm}=5 / 100 \mathrm{~B}$,

donne $\mathrm{B}$ de l'ordre de 6 à $10 \mathrm{~cm}$.

Pour des largeurs plus petites que ces valeurs, la surface de rupture se développe en même temps que la rupture générale de la fondation: le travail de dilatance et le travail de rupture générale sont simultanés et la portance est élevée. Pour des largeurs plus grandes, la surface de glissement se développe en commençant à partir du bord de la fondation dès que l'enfoncement $w$ dépasse $3 \mathrm{~mm}$ et le mécanisme de rupture progressive entraîne la séparation entre le travail de la dilatance et le travail de frottement: le travail de la dilatance est concentré dans une bande située à l'avant de la surface de glissement et se déplace avec elle au fur et à mesure qu'elle avance; plus la surface de glissement est longue plus le travail de dilatance est a dilué » dans le travail de poinçonnement: la portance paraît plus faible puisque l'effet de la dilatance diminue. $B=10 \mathrm{~cm}$ apparaît donc comme une valeur en deçà de laquelle l'effet d'échelle est important, au-delà de laquelle il s'estompe et disparaît. La figure 3, d'après TCHENG et ISEUX, (1966), donne la valeur de $\mathrm{N}$, en fonction de la largeur $\mathrm{B}$ de la fondation pour des essais sur sables denses. La largeur critique qu'on peut en déduire est de 10 à $12 \mathrm{~cm}$, en accord avec l'évaluation précédente. La figure 4, d'après AMAR et al., (1984), donne une largeur critique du même ordre de grandeur.

On remarquera que les coefficients $N_{\gamma}$ des petites fondations correspondent à des angles de frottement interne très grands. Ils devraient évidemment être associés aux valeurs maximales des courbes effortdéformation des essais de caractérisation mécanique.

\section{COMMENTAIRES}

\section{Effet d'échelle sur les argiles}

L'existence d'un effet d'échelle pour des essais en modèles réduits sur des argiles ne semble pas avoir été signalée. On pourrait dire dans le cadre de la même

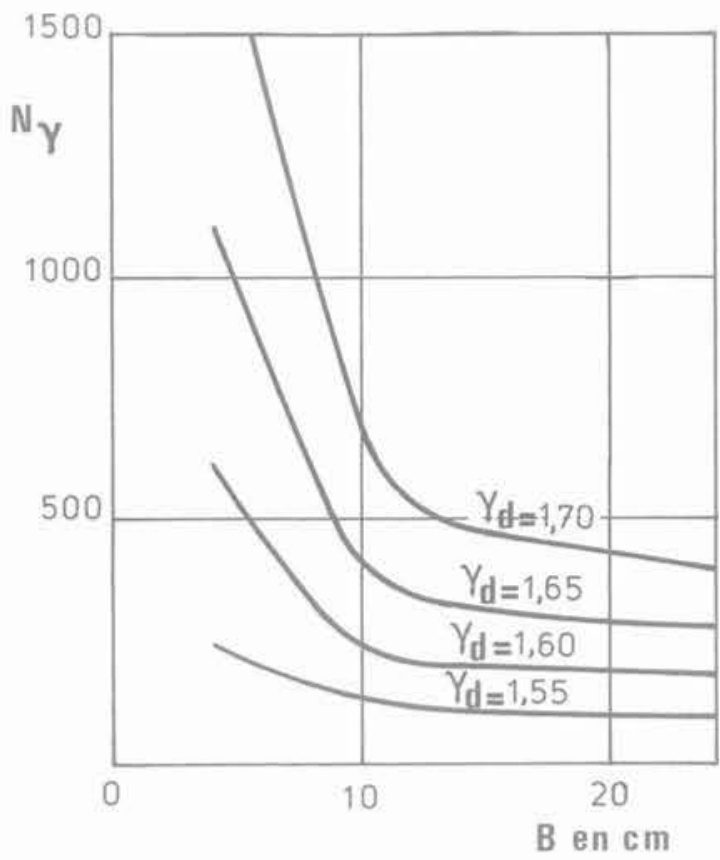

Fig. 3. - Variation de $N_{\gamma}$ en fonction de la largeur $B$ de la fondation (sable de Loire) (d'après Tcheng et Yseux, 1966).

interprétation que les grains d'argile sont tellement petits que la dilatance y est pratiquement nulle et que le travail contre la pesanteur engendré par la variation du volume du sol en cours de rupture est insignifiant. Cette interprétation est insuffisante. II faut d'abord remarquer que les essais sur modèles réduits ont pratiquement toujours été faits sur de l'argile relativement molle ou sur de la pâte à modeler, c'est-à-dire sur des matériaux qui présentent de l'écrouissage. Dans ces conditions (P. HABIB, 1984), il n'y a pas de formation de surfaces de glissement. Les surfaces de glissement n'apparaissent qu'avec des argiles raides à comportement radoucissant. Dans un tel cas, pour des fondations assez grandes, un mécanisme de rupture progressive peut se produire. L'observation courante des essais de cisaillement rectiligne ou des essais 


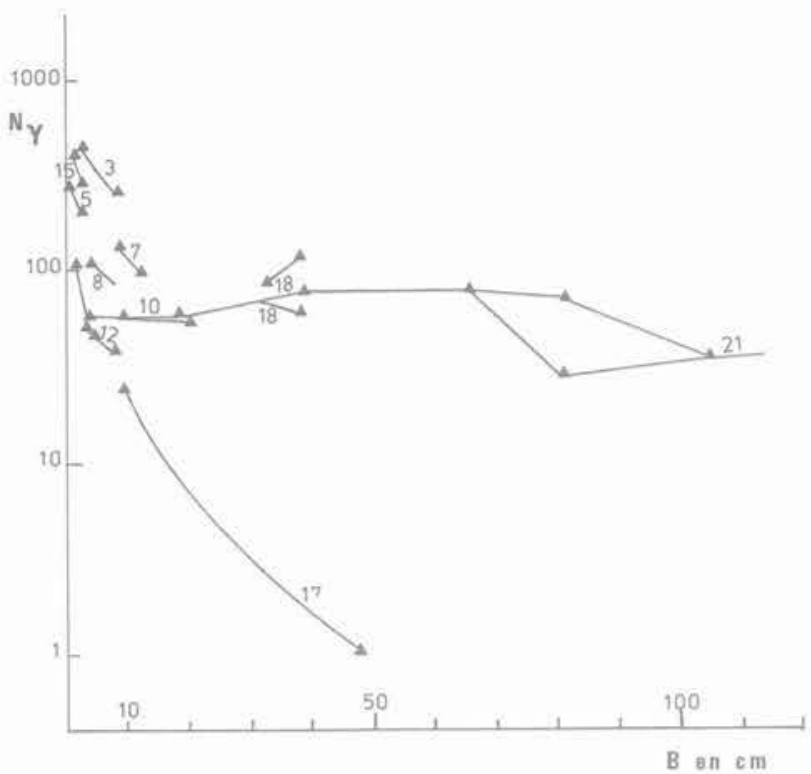

Fig. 4. - Variations de $N_{\gamma}$ en fonction de la largeur $B$ de la fondation, obtenues par différents auteurs (d'après Amar et al., 1984).

triaxiaux sur argile montre que la surface de glissement apparaît après un déplacement relatif de l'ordre de 3 à $5 \mathrm{~mm}$ des parties situées de part et d'autre de la discontinuité, c'est-à-dire à peu près la même chose que pour les sables. Il en résulte à nouveau que la rupture progressive peut se produire et par conséquent qu'un effet d'échelle doit exister lorsque la largeur B est plus petite que $10 \mathrm{~cm}$ pour des fondations à la surface d'un massif d'argile radoucissante.

Pourtant l'effet d'échelle n'a pas été signalé pour des essais en modèles réduits sur argile en laboratoire. L'explication en est simple. Pour une argile radoucissante avec $\phi_{u \text { u }}=0$, il faut associer deux valeurs de la cohésion $C_{\max }$ et $C_{\text {residuelle, et ces valeurs ne sont pas }}$ très différentes lorsque la consolidation de l'argile est moyenne. Or, la force portante $\mathrm{CN}_{\mathrm{c}}$ est proportionnelle à la cohésion, ce quí fait que la diminution de portance due à la rupture progressive peut passer inaperçue alors que pour les sables un écart modeste entre $\phi_{\max }$ et $\phi_{r}$ entraîne une grande différence entre les portances à cause de l'effet multiplicateur du frottement, différence que l'on retrouve par exemple, en comparant les expressions théoriques de $\mathrm{N}_{\gamma}\left(\phi_{\max }\right)$ et $N_{y}\left(\phi_{r}\right)$.

Ainsi, pour que l'effet d'échelle sur une argile soit sensible, il faut que le rapport $C_{\max } / C_{r}$ soit grand. Cela ne peut se produire que pour des argiles très fortement consolidées. Mais alors la préparation d'un grand bloc d'un tel matériau pour un modèle réduit un peu grand, nécessite l'application pendant un temps très long d'une force considérable. Par exemple, pour consolider sous $0,8 \mathrm{MPa}$ un bloc de $30 \mathrm{~cm}$ d'épaisseur et de $90 \mathrm{~cm}$ de diamètre, juste suffisant pour étudier un poinçon circulaire de $30 \mathrm{~cm}$ de diamètre, il faut exercer une force de $500 \mathrm{kN}$ pendant plusieurs mois. Il ne faut donc pas s'étonner si de tels essais n'ont pas été réalisés systématiquement.

\section{Effet d'échelle sur les sables lâches}

Avec l'interprétation précédente et puisqu'il ne se forme pas de surface de glissement isolée dans un sable lâche, les conditions de similitude sont vérifiées si les lois de comportement du sol sont les mêmes sous deux fondations homothétiques. Cette condition est a priori vérifiée si le sable est le même sous une petite et sous une grande fondation superficielle.

L'appréciation de la charge ultime est évidemment difficile lorsque le matériau est écrouissable, d'une part parce que la rupture sous une fondation superficielle n'est pas nette avec un sable lâche, mais aussi parce que dans un sable, la portance augmente avec l'enfoncement Il n'en reste pas moins que l'interprétation proposée ici entraîne l'absence d'effet d'échelle pour les modèles réduits de fondations sur des milieux pulvérulents lâches. A ma connaissance, un tel effet n'a jamais été signalé.

\section{Cas des enrochements}

Sì l'on considère un matériau d'enrochements comme un milieu pulvérulent, il n'y a pas de différence entre un massif de sable et un massif d'enrochements, hormis la dimension des grains.

On a vu précédemment qu'une surface de glissement se forme dans un sable dont le grain moyen est de l'ordre de $0,5 \mathrm{~mm}$ après un déplacement relatif de 3 à $5 \mathrm{~mm}$; on peut dire aussi qu'un déplacement relatif d'une dizaine de grains est nécessaire pour provoquer le désenchevêtrement correspondant à l'apparition d'une surface de glissement. $\mathrm{Ce}$ critère géométrique appliqué à des enrochements de $30 \mathrm{~cm}$ de grain moyen montre qu'il faudrait un déplacement relatif de $3 \mathrm{~m}$ pour faire apparaître une surface de glissement dans un massif d'enrochements: autant dire qu'on n'en voit jamais. Tout au plus sur un talus d'enrochements trop incliné, voit-on quelques blocs isolés instables rouler jusqu'à la base mais sans que cela produise une rupture franche avec l'apparition d'une surface de glissement.

En utilisant la même règle géométrique, on peut rapprocher l'effet d'échelle et la dimension des grains: on peut dire par exemple que l'effet d'échelle sur les matériaux pulvérulents ne se produit que pour des fondations dont la largeur est inférieure à 200 grains. Pour une fondation sur un massif d'enrochements, cela signifie que la largeur critique à partir de laquelle une rupture progressive serait à craindre est de l'ordre de 60 à $100 \mathrm{~m}$. Cela signifie aussi que cette dimension est un ordre de grandeur d'une taille critique pour une structure: plus petite, elle peut et doit être calculée avec $\varphi_{\max }$, plus grande il faut être prudent. Ainsi, un talus en enrochements de 60 à $100 \mathrm{~m}$ de haut est certainement extrêmement résistant, beaucoup plus même qu'on ne le croit généralement; par contre, pour des hauteurs nettement plus grandes, 250 ou $300 \mathrm{~m}$, il est sans doute imprudent d'extrapoler l'expérience acquise à partir de talus de 60 à $100 \mathrm{~m}$ de haut et de construire des talus très raides, sauf à prendre des blocs plus gros. Mais, quel est l'angle de talus naturel d'un massif d'enrochements ou celui d'un massif de sable? 


\section{Angle de talus naturel d'un sable}

L'angle $\alpha$ à la base d'un talus naturel en équilibre limite est théoriquement égal à l'angle de frottement interne $\phi$ du critère de Coulomb. Pourtant, ce phénomène n'est jamais utilisé actuellement comme expérience pour déterminer l'angle de frottement interne d'un sable. Il y a pour cela de nombreuses raisons. Par exemple, la petite cohésion engendrée par un peu d'humidité dans une masse de sable peut devenir une source d'erreur considérable. Mais, même pour un sable parfaitement sec, il reste des incertitudes et on constate notamment l'existence de deux angles de repos: l'angle de talus en remblai, voisin d'une trentaine de degrés pour les sables courants et l'angle de talus en déblai, qui est un peu plus grand, probablement à cause du caractère dynamique de la mise en place d'un sable par remblai et évidemment aussi du fait qu'un sable déversé est toujours un sable lâche. On constate cependant que le tas conique formé par un sable déversé soigneusement sur un plateau mobile (fig. 5) supporte une légère inclinaison supplémentaire, de 3 à $4^{\circ}$, avant de glisser; si on gratte délicatement au pied de ce même talus en remblai, on peut obtenir un angle un peu plus grand pour atteindre la pente du déblai.

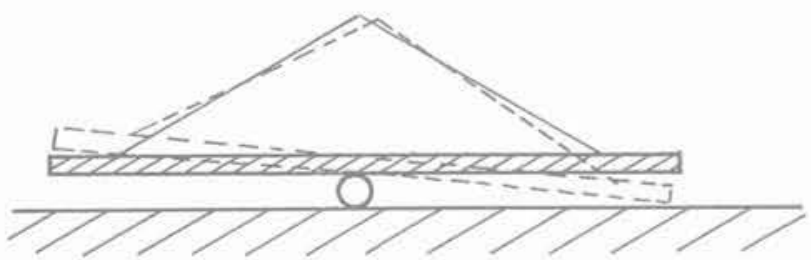

Fig. 5. - Dispositif expérimental pour l'étude de la stabilité d'un talus conique en remblai en fonction de l'inclinaison de son support.

Les angles de déblai sont évidemment beaucoup plus grands lorsqu'on creuse au pied d'un massif de sable dense; mais, on constate alors que l'effondrement ne se produit pas par un mécanisme de glissement circulaire profond mais pas des avalanches régressives superficielles: d'ailleurs, le fourmi-lion qui attend au fond de son piège en entonnoir taillé à l'angle de déblai, sait bien qu'il ne risque pas d'être emporté par un glissement de terrain profond, mais que sa proie va glisser gentiment jusqu'à lui. De même, si on incline un massif de sable dense, la rupture de la pente se produit par l'avalanche déclenchée par le premier grain de la surface qui perd l'équilibre puis roule et entraîne les grains suivants: pour enrayer ce mécanisme, il suffirait donc d'empêcher le premier grain de bouger. Pour obtenir ce résultat, nous avons réalisé l'expé. rience suivante. Un sable de Fontainebleau (grains moyens $0,2 \mathrm{~mm}$ ) a été vibré dans un récipient puis le remplissage a été terminé par saupoudrage; la densité globale était $\gamma=1,61$ (alors que la densité du sable déversé était de 1,41). Sur la surface du massif de sable, on a laissé se condenser le léger brouillard d'un aérosol d'eau, puis on a incliné le récipient contenant le sable jusqu'à la rupture. L'inclinaison de la surface du sable a atteint $51^{\circ}$ (fig. 6). L'effondrement du sable

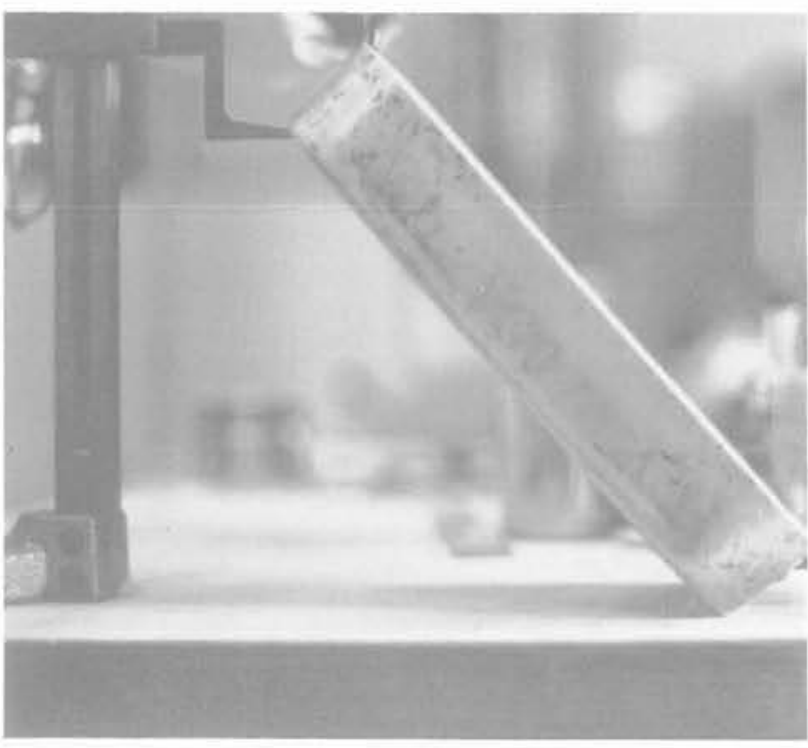

Fig. 6. - Dispositif expérimental pour l'étude de l'angle de talus naturel d'un massif de sable dont la surface a été fixée. Longueur de la cuve: $30 \mathrm{~cm}$, largeur de la cuve: $20 \mathrm{~cm}$.

Photographie prise juste avant la rupture.

s'est produit très brutalement (fig. 7). Dans le cas du poinçon superficiel, on peut s'attendre à une rupture progressive sous une fondation large parce que le gradient de contrainte est particulièrement grand: le critère de rupture est d'abord atteint près du bord de la fondation puis la plastification se propage. Pour un

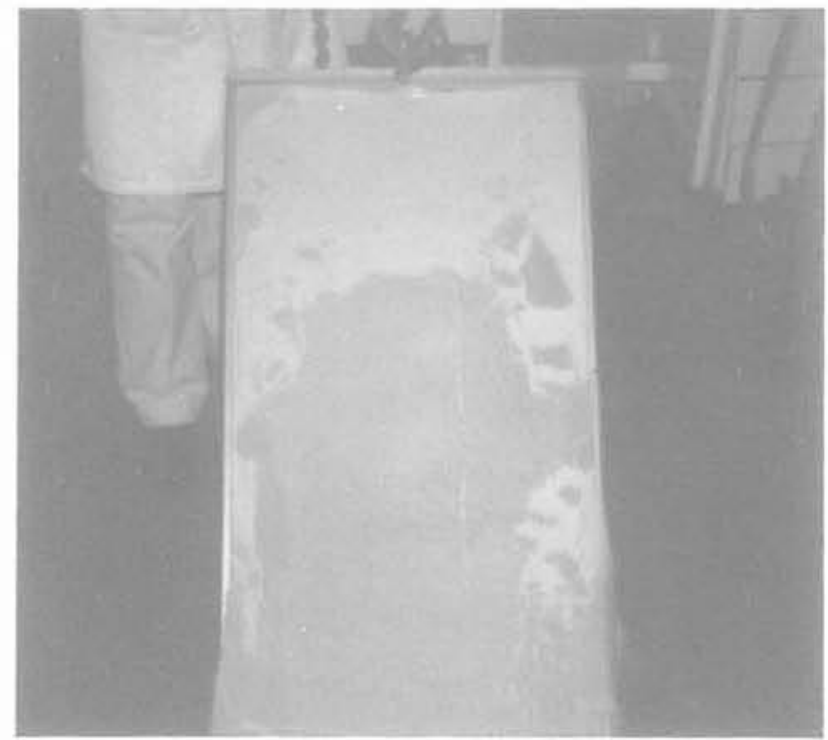

Fig. 7. - Photographie prise pendant la rupture générale du talus.

Les parties sombres correspondent à la surface humidifiée, les déchirures blanches au sable sec sous-jacent. Ce glissement général immédiatement sous la "peau" est bien conforme au schéma du calcul à la rupture.

Longueur de la cuve: $97 \mathrm{~cm}$, largeur de la cuve: $49 \mathrm{~cm}$. 
massif de sable mis en place à l'horizontale puis incliné, le gradient de contrainte, parallèlement à la surface libre, est pratiquement nul ou plutôt si le sable est homogène, le critère de rupture est atteint à peu près partout en même temps dans le massif. L'effondrement est donc tout à fait soudain et il ne devrait pas y avoir d'effet d'échelle puisque le champ de rupture est homogène. On voit sur la figure 8 que le sable était parfaitement pulvérulent; de toute façon, même si on peut dire que des liaisons capillaires donnaient une certaine cohésion entre les grains de la surface, le plan immédiatement en-dessous était entièrement dans du sable sec, c'est-à-dire sans cohésion. L'angle de frottement interne d'un sable sec peut alors atteindre de très grandes valeurs supérieures à $45^{\circ}-$ la plus grande inclinaison que nous avons mesurée a atteint $51^{\circ}$ - des valeurs qu'on accepte habituellement uniquement pour les enrochements.

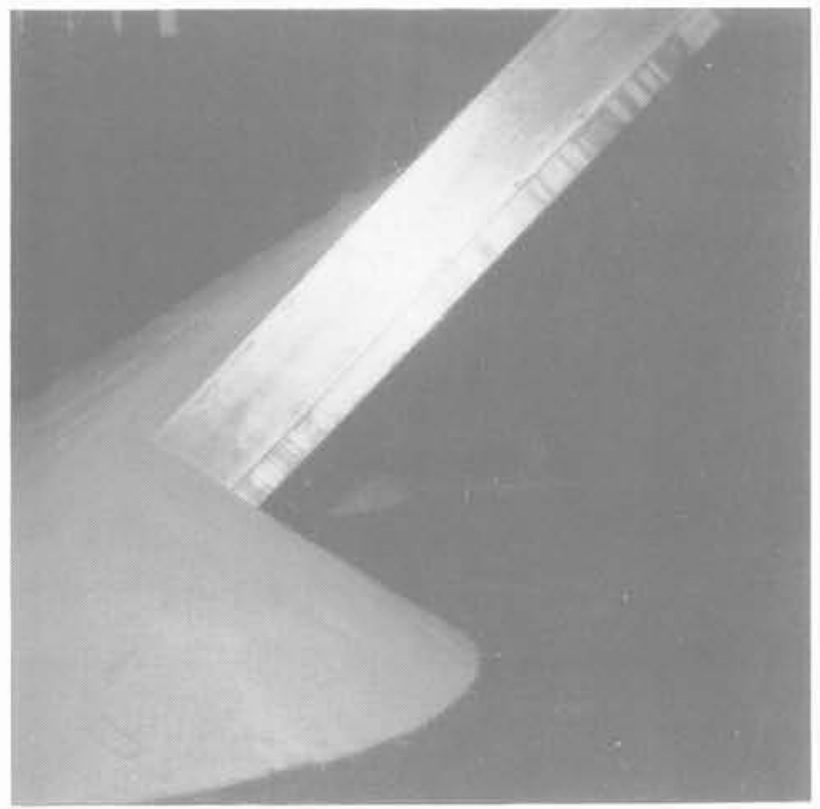

Fig. 8. - Photographie du dispositif après la rupture générale.

L'angle de talus du déblai est voisin de $30^{\circ}$ (sable de Fontainebleau).

Pourtant la mise en place d'un sable et son serrage paraissent très différents de ceux des enrochements. En particulier, le compactage d'un sable n'est pas aussi efficace que celui d'un enrochement où l'on arrive à imbriquer les grains très étroitement au point de pouvoir ensuite $y$ tailler un talus â front très raide presque vertical. En fait, la comparaison n'est pas complète et si l'on veut respecter la similitude entre un sable et un enrochement, il faut que l'échelle des résistances des grains, c'est-à-dire l'échelle des contraintes, soit égale à l'échelle des longueurs. Ainsi, l'équivalent d'un enrochement en bon granite est un sable composé de grains d'une très mauvaise craie: il est alors tout à fait intuitif qu'un tel milieu pulvérulent pourrait être facilement compacté jusqu'à prendre l'apparence d'un milieu cohérent et pouvoir être taillé à front raide.

\section{CONCLUSION}

Le développement progressif d'une surface de glissement dans les sables denses avec localisation de la dilatance est responsable de l'effet d'échelle que l'on constate pour les poinçons de moins de $15 \mathrm{~cm}$ de largeur. Le coefficient $\mathrm{N}_{\text {, }}$ associé aux modèles les plus réduits est parfois très grand; il correspond à de grandes valeurs de l'angle de frottement interne. Il n'y a pas d'effet d'échelle pour les sables lâches ni pour les argiles raisonnablement plastiques. Il est probable qu'il existe un effet d'échelle pour des poinconnements sur des argiles très raides même non fissurées.

Des angles de frottement interne extrêmement grands peuvent être mis facilement en évidence sur des sables secs dans le champ de contraintes très régulier correspondant à l'équilibre de Rankine; ils expliquent les très grandes valeurs de $\mathrm{N}_{\gamma}$ observées avec les tous petits poinçons. Le même concept géométrique appliqué aux enrochements montre que la sécurité d'un talus en enrochement, de 60 à $100 \mathrm{~m}$ de haut, est probablement très grande mais que l'extrapolation à de plus grands ouvrages doit être faite avec prudence.

L'auteur tient à remercier Pierre LONDE des conversations extrêmement fructueuses qu'il a eues avec lui à l'occasion de la rédaction de cet article.

\section{BIBLIOGRAPHIE}

AMAR S.; BAGUELIN F.; CANEPA Y. (1983). Etude expérimentale du comportement des fondations superficielles, Annales I.T.B.T.P., $n^{\circ} 427$. sept. 1984, pp. 83-109.

DE BEER E.E. (1965). - The Scale Effect on the Phenomenon of progressive Rupture in cohesionless Soils, C.R. $6^{e}$ Conf. Int. Mec. Sol. Montréal Canada, vol. II, pp. 13-17.

HABIB P. (1961). - Force portante et déformation des fondations superficielles, Annales I.T.B.T.P., juil-août 1961, pp. 759-772.

HABIB P. (1973). - Effet d'échelle sur les sables denses, séminaire «Plasticité et Viscoplasticité 1972 *. Sciences et Techniques de l'Armement, Paris, $2^{e}$ fascicule, 1973 , pp. 501-506.

HABIB P. (1984). - Les surfaces de glissement en mécanique des sols, Revue Française de Géotechnique, $\mathrm{n}^{\circ} 27$, pp. 7-21.

TCHENG Y.; ISEUX J. (1966). - Nouvelles recherches sur le pouvoir portant des milieux pulvérulents: fondations superficielles et semi-profondes, Annales I.T.B.T.P., $\mathrm{n}^{\circ} 227$, Paris, nov. 1966, pp. $1267-1282$. 